\title{
Differential regulation of brain-derived neurotrophic factor transcripts during the consolidation of fear learning
}

\author{
Lisa M. Rattiner, Michael Davis, and Kerry J. Ressler ${ }^{1}$ \\ Emory University School of Medicine, Center for Behavioral Neuroscience, Yerkes Research Center, Atlanta, Georgia 30329, USA
}

\begin{abstract}
Brain-derived neurotrophic factor (BDNF) has been implicated as a molecular mediator of learning and memory. The BDNF gene contains four differentially regulated promoters that generate four distinct mRNA transcripts, each containing a unique noncoding $5^{\prime}$-exon and a common $3^{\prime}$-coding exon. This study describes novel evidence for the differential usage of alternative BDNF promoters and 5 '-exons during the consolidation of learning. We found a selective increase in BDNF transcripts containing exons I and III in the amygdala $2 \mathrm{~h}$ following fear conditioning, while mRNA levels of BDNF exons II and IV remained unchanged. These results provide the first evidence of differential splicing and/or differential BDNF promoter usage in response to a behaviorally relevant learning paradigm.
\end{abstract}

In addition to its trophic function, brain-derived neurotrophic factor (BDNF) has been implicated in synaptic plasticity and learning and memory. BDNF has been shown to play a critical role in the induction and maintenance of long-term potentiation as well as in hippocampal-dependent learning (Korte et al. 1995; Figurov et al. 1996; Kang et al. 1997; Berchtold et al. 1999; Hall et al. 2000; Poo 2001). More recently, we have found a role for BDNF in amygdala-dependent fear conditioning (Rattiner et al. 2004). Despite increasing interest in this gene for studying the molecular mechanisms underlying learning and memory, the transcriptional regulation of the BDNF gene during learning and memory processes has yet to be closely examined. The BDNF gene has four distinct transcripts, each containing a unique promoter. These BDNF transcripts consist of a variable $5^{\prime}$-exon (exons I-IV) and a common 3'-exon (exon V). The common 3'-exon codes for the mature BDNF protein, whereas the untranslated exons I-IV arise from differential utilization of the four promoters (Timmusk et al. 1993). The existence of multiple BDNF mRNA transcripts that are all translated into an identical protein is a unique feature of this neurotrophin that suggests multilevel regulation of its expression.

The multiple BDNF promoters are likely recruited to generate region- or activity-specific expression of the different BDNF transcripts and are differentially sensitive to a variety of stimuli (Bishop et al. 1994; Kokaia et al. 1994). It has been shown that specific exon-containing BDNF mRNAs are differentially regulated by interventions such as exercise, seizures, ischemia, osmotic stress, and antidepressant treatment (Lindvall et al. 1992; Kokaia et al. 1994; Oliff et al. 1998; Aliaga et al. 2002; Dias et al. 2003). However, no studies to date have demonstrated whether the BDNF promoters are differentially activated during behaviorally relevant learning paradigms.

It is well established that cue-specific fear conditioning is dependent on the basolateral amygdala (BLA) (Davis et al. 1993; Fanselow and LeDoux 1999). We have recently reported an upregulation of BDNF mRNA in the amygdala following this type of fear conditioning (Rattiner et al. 2004). In this report, we exam-

\footnotetext{
'Corresponding author.

E-mail kressle@emory.edu; fax (404) 727-8070.

Article published online ahead of print. Article and publication date are at http://www.learnmem.org/cgi/doi/10.1101//m.83304.
}

ine whether use of these multiple promoters directs activityspecific expression of different BDNF transcripts within the BLA following Pavlovian fear conditioning.

Preliminary experiments examined whether long-standing regulatory changes in BDNF occurred following fear training. This experiment allowed us to determine the optimal time point to observe changes in BDNF gene expression. A total of 32 male Sprague-Dawley rats (Charles River, Raleigh, NC) were trained with either 15 light-shock pairings $(n=18)$ or 15 light-alone presentations $(n=18)$. Training, testing, and analysis of the acoustic startle reflex took place as described previously (Cassella et al. 1986; Walker and Davis 2000; Paschall and Davis 2002). The unconditioned stimulus (US) was a 0.5 -sec, $0.4-\mathrm{mA}$ footshock delivered through the cage floor bars. The conditioned stimulus (CS) for training and testing was a 4-sec light (82 lux) produced by an 8 -W fluorescent bulb located $10 \mathrm{~cm}$ behind each cage.

Animals were returned to their home cages after training. Then 12 animals were killed $2 \mathrm{~h}$ after training ( $n=6$ light-shock, $n=6$ light alone), 10 animals were killed $24 \mathrm{~h}$ later $(n=6$ lightshock, $n=4$ light alone), and 10 animals were killed $7 \mathrm{~d}$ later ( $n=6$ light-shock, $n=4$ light alone) for in situ hybridization analysis. In situ hybridization was performed with antisense riboprobes. A partial BDNF clone, containing the entire exon $\mathrm{V}$ coding sequence but no significant portion of a $5^{\prime}$-exon, was obtained from the NIH database (IMAGE \#1851120, GenBank \#3837569).

In situ hybridization was performed using 35S-UTP (NEN) labeled riboprobes prepared from linearized clones with RNA polymerase. Hybridizations were performed under parafilm overnight at $52^{\circ} \mathrm{C}$ as described (Ressler et al. 2002). After hybridization, slides were stringently washed, dried, and placed against Kodak "maximum resolution" autoradiography film for $5 \mathrm{~d}$.

Figure 1A shows significant increases in BDNF exon $\mathrm{V}$ mRNA at $2 \mathrm{~h}$ in light-shock-trained animals compared with all other data points $\left(F_{5,31}=4.0, p=0.008\right)$. Post hoc analysis showed significant differences between the light-shock and light-alone groups at $2 \mathrm{~h}(p=0.001)$, but not between these groups at $24 \mathrm{~h}(p=0.43)$ or $7 \mathrm{~d}(p=0.32)$. These data suggest that $\mathrm{BDNF}$ is rapidly regulated during the consolidation period following fear conditioning, but the gene expression changes are not maintained. Therefore, BDNF gene expression was examined at $2 \mathrm{~h}$ following training for subsequent studies. 
A

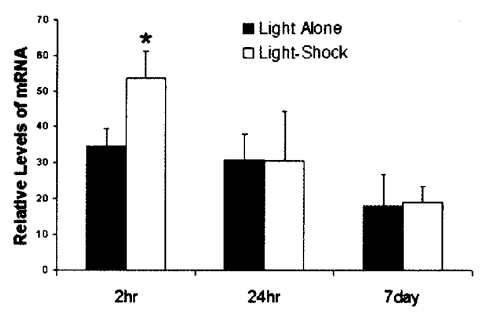

B

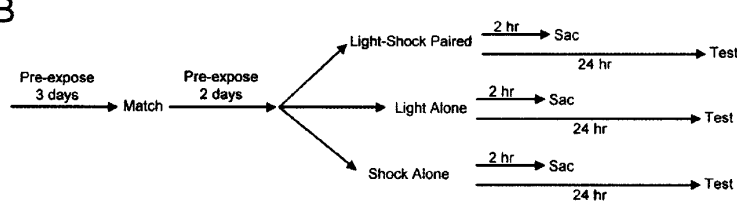

C

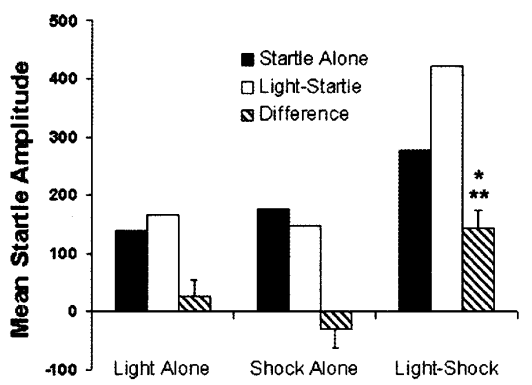

D

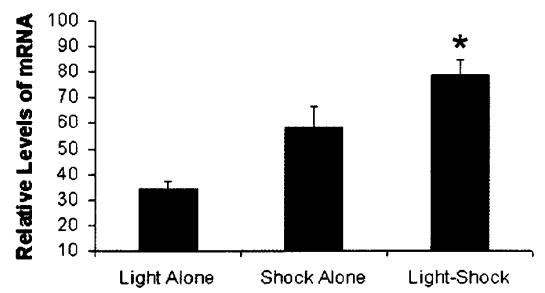

Figure 1. $B D N F$ exon $V$ in situ hybridization and results of behavioral testing. $(A)$ Levels of BDNF exon V mRNA in the BLA at different time points following light-shock or light-alone presentations. There is a significant increase in BDNF exon $\mathrm{V}$ mRNA at $2 \mathrm{~h}$ in light-shock-trained animals compared with all other data points ( ${ }^{*}$ ANOVA; $p<0.05$ ). Post hoc analysis showed significant differences between the light-shock and light-alone groups at $2 \mathrm{~h}$, but not between these groups at $24 \mathrm{~h}$ or $7 \mathrm{~d}$. $(B)$ Animals were exposed to either light-alone or shock-alone presentations, or associative light-shock pairings. Animals were either killed $2 \mathrm{~h}$ later or tested $24 \mathrm{~h}$ later for the presence of fear-potentiated startle. (C) Light-shock pairings produced stable fear memories in the light-shock associative group, as assessed by the fear-potentiated startle effect (*Paired $t$-test; $p<0.05$ ). Light-alone and shock-alone control groups show no appreciable difference between startle in the absence or presence of the light. The difference scores for the light-shock group are significantly different from the difference scores for the light-alone or shock-alone controls ( ${ }^{* *}$ ANOVA; $p<0.05$ ). (D) Levels of exon $V$ are significantly increased in the BLA after light-shock fear conditioning when compared with light-alone or shock-alone control groups ( ${ }^{\star}$ ANOVA; $p \leq 0.05$ ). The exon V-specific transcript is common to all BDNF splice variants, thus the exon $\mathrm{V}$ in situ probe recognizes all full-length transcripts regardless of the $5^{\prime}$-exon used.

The statistics above (Fig. 1A) were obtained with a conventional method of analyzing in situ hybridization and receptor binding autoradiography using the AIS technique calibrated with ${ }^{14} \mathrm{C}$-labeled standards (AIS system, Imaging Research) (Liu et al. 2001; Poulsen et al. 2004). However, we have found that scan- ning the autoradiography films into a PC allows for better future archival access and cataloging of the data. Furthermore, we have found that analysis of these digital images using ADOBE Photoshop produces a more conservative and easier analysis technique.

To validate this Digital Photoshop method, we repeated the analysis of the same films. Films were first scanned into a PC at 600 dpi. Hybridization density quantification was performed with the mean luminosity histogram feature of Adobe Photoshop at a level of exposure that produced linear densities with ${ }^{14} \mathrm{C}$ standards. Within each experiment, all slides hybridized to the same probe were exposed to the same piece of film for the same amount of time. This ensured equivalent exposure times and conditions between animals and experimental groups. For each section, hybridization density was determined for the regions of interest (ROI), as well as an adjacent background area that lacks hybridization (e.g., internal capsule). Normalized density was determined as previously described (Ressler et al. 2002; Rattiner et al. 2004). For each experimental group, hybridization density is reported as the average density of all individual animals for that condition \pm SEM. Comparison of means between experimental groups was performed with ANOVA followed by a post hoc Tukeys test using the SPSS statistics package.

Blinded analysis of this same data set leads to identical results with the Digital Photoshop method. There is a significant increase in exon $\mathrm{V}$ at $2 \mathrm{~h}$ in fear-trained animals compared with all other data points $\left(F_{5,31}=4.2, p=0.006\right)$. Post hoc analysis showed significant differences between the light-shock and light-alone groups at $2 \mathrm{~h}(p=0.03)$, but not between these groups at $24 \mathrm{~h}(p=0.98)$ or $7 \mathrm{~d}(p=0.90)$. Linear regression demonstrated a highly significant correlation between the AIS and Digital Photoshop generated data points across all animals within this experiment $\left(F_{1,31}=16.8, p<0.001, R=0.60\right)$. Therefore, because of the advantages listed above for the Digital Photoshop method, we used this method for analysis of the remaining in situ hybridization experiments.

Our next series of experiments examined the differential regulation of BDNF transcripts. A total of 28 male SpragueDawley rats (Charles River, Raleigh, NC) were trained and tested as shown in Figure 1B. Training and testing procedures occurred as described above. All animals were pre-exposed to handling and placement in the training/testing chambers for $5 \mathrm{~d}$ prior to fear conditioning. On day 3 of pre-exposure, baseline startle was measured by presenting 30 startle stimuli at a 30 -sec interstimulus interval (ISI). On the basis of these results, animals were divided into matched experimental groups with equivalent mean startle amplitudes. On training day, animals received 15 light-shock pairings (light-shock pairing; $n=10$ ), 15 light presentations alone (light-alone control; $n=9$ ), or 15 shock presentations alone (shock-alone control; $n=9$ ) and were returned to their home cage. Light-shock pairings were given with an average ISI of $2 \mathrm{~min}$, creating a 30-min training period. The shock (US) was delivered during the last $0.5 \mathrm{sec}$ and coterminated with the 4-sec light (CS). The light-alone control group received 154 -sec light presentations, with an average ISI of 2 min, over a 30-min training period. The shock-alone group received 150.5 -sec shocks, with an average ISI of 2 min (range, 3-5 min), over a 30-min training session.

Animals from all groups were returned to home cages after training. Then, 16 animals were killed $2 \mathrm{~h}$ later for in situ hybridization ( $n=6$ light-shock, $n=5$ shock alone, $n=5$ light alone). Another 12 animals were kept for behavioral testing $24 \mathrm{~h}$ later ( $n=4$ in each of the three groups). During fear-potentiated startle testing, animals are given a 5-min acclimation period followed by 30 startle stimuli $(95 \mathrm{~dB}$ ) with a 30 -sec ISI (leader stimuli), followed by 30 startle-alone test trials and 30 intermixed light-startle test trials. 
Animals that had experienced light-shock pairings showed significant fear-potentiated startle (Fig. 1C; Paired $t$-test; ${ }^{*} p=0.01$ ). Animals that had received light-alone or shock-alone training showed no appreciable difference between startle in the presence or absence of light. The difference scores were also significantly different for the light-shock-trained group compared with control groups (Fig. 1C; ANOVA: $F_{(2,9)}=11.5,{ }^{* *} p=0.003$ ).

Animals ( $n=6$ light-shock, $n=5$ shock alone, $n=5$ light alone) were killed $2 \mathrm{~h}$ after fear conditioning, and brains were prepared for in situ hybridization analysis. We found significant increases in the light-shock paired group compared with controls with the exon V-specific mRNA (Fig. 1D; $F_{2,10}=20.4 ; p=0.048$ ). This replicated our original finding that BDNF mRNA levels are elevated in the amygdala $2 \mathrm{~h}$ following fear conditioning (Fig. 1A).

This result was expected because the exon $\mathrm{V}$ probe will recognize the entire pool of BDNF transcripts. For specific exon analyses, exons I-IV were PCR-amplified from larger BDNF clones (obtained from Bradley Pearce and J.C. Lauterborn, University of California, Irvine), subcloned into the pCRII-TOPO vector, and sequence-verified. Figure $2 \mathrm{~A}$ illustrates the regions within these exons used for in situ analysis. In situ hybridization was performed with antisense riboprobes, and films were analyzed using the Digital Photoshop method described.

Prior to examining the regulation of the four different $5^{\prime}$ transcripts, we demonstrated the specificity of the individual probes using a series of in situ "cold-probe" competition experiments. Following previously accepted techniques, we produced nonradioactive RNAs for each sequence-verified exon (Remick et al. 1988; Pelletier et al. 1992). We then hybridized combinations of all nonradioactive RNA transcripts with each radiolabeled probe, yielding 16 different combinations of hot and cold probes: for example, cold exon I probe with ${ }^{35} \mathrm{~S}$-exon I probe (I-I), cold exon I probe with ${ }^{35} \mathrm{~S}$-exon II probe (I-II), cold exon I probe with ${ }^{35}$ S-exon III probe (I-III), and so on. In situ hybridization was performed as described above. All slides were prehybridized with cold probe for $5 \mathrm{~h}$, washed with $5 \times$ SSC, and then cohybridized

A

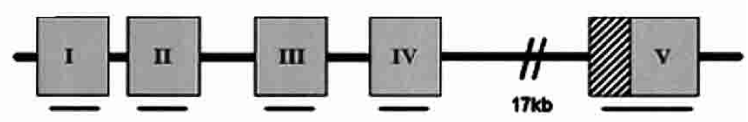

B

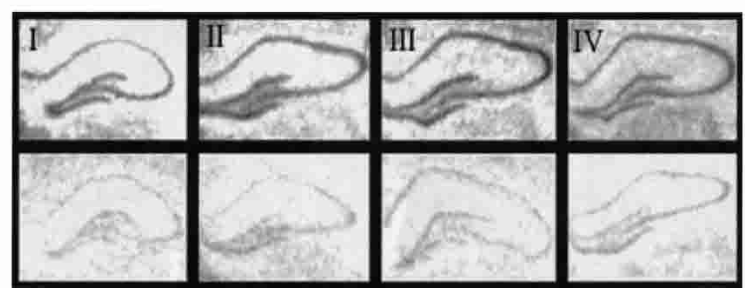

Figure 2. In situ hybridization cold-probe competition experiment. $(A)$ Genomic structure of the rat BDNF gene. Exons are indicated by boxes, and introns by lines. Filled boxes indicate untranslated mRNA. The hatched portion of exon $\mathrm{V}$ indicates the coding region of the BDNF gene. The rat $B D N F$ gene is transcribed from four different promoters, which immediately precede each of the first four exons. Therefore each fulllength transcript contains a unique 5 '-exon (I-IV) and a common 3 '-exon (exon $V$ ) that encodes the mature protein. The lines below each exon correspond to the region of each exon used to make in situ hybridization probes. (B) In situ hybridization analysis of BDNF exons I-IV in the hippocampus. Representative sections are shown in which a specific exon probe was cohybridized with its cold counterpart (bottom row), or in which the specific exon probe was cohybridized with a cold probe of a different exon (top row).

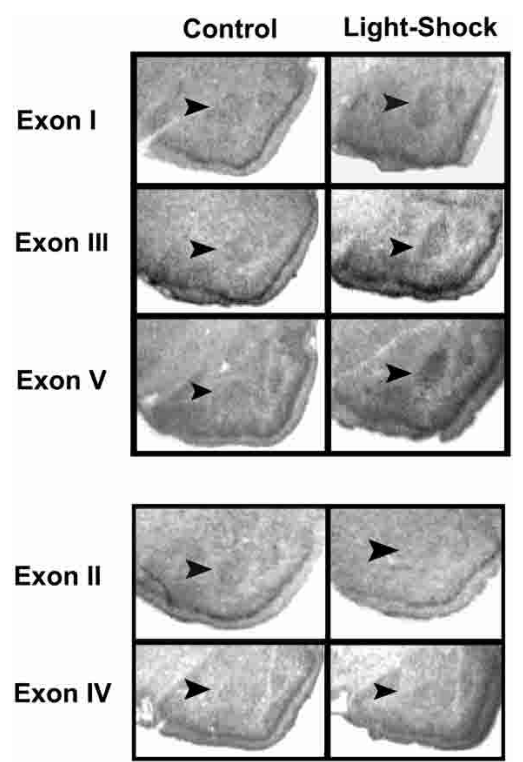

Figure 3. In situ hybridization using probes for different BDNF transcripts. In situ hybridization analysis of BDNF transcripts in the BLA following light-alone presentation (control) and light-shock pairings. Magnified images are autoradiographic images of BLA sections following hybridization with ${ }^{35} \mathrm{~S}$-radiolabeled probes for BDNF transcripts.

overnight with ${ }^{35}$ S-probe and the nonradioactive cold probe. Figure $2 \mathrm{~B}$ shows representative hippocampal sections (where there is substantial and stable expression of the four exons) of a specific exon probe cohybridized with its cold counterpart (Fig. 2B, bottom row), as well as the specific exon probe cohybridized with a cold probe of a different exon (Fig. 2B, top row). We found a significant loss in binding of the ${ }^{35} \mathrm{~S}$-probe after competition with its cold-labeled counterpart (e.g., I-I, II-II, III-III, IV-IV), but no significant competition with cold probes of any other exons. This experiment demonstrates that each antisense riboprobe used in the subsequent experiment is specific for each $5^{\prime}$ untranslated BDNF exon described, and essentially no nonspecific labeling occurs with these probes.

Tissue sections obtained from the light-shock experiment described above were processed for in situ hybridization using our exon-specific probes. Figure 3 illustrates that there are basal levels of all BDNF transcripts in the amygdala (control); however, following fear conditioning (light-shock), hybridization was markedly increased in the amygdala for exon I, III, and V transcripts.

Upon quantitative examination of the individual BDNF transcripts, we found that exon I and III transcripts were significantly increased in the BLA $2 \mathrm{~h}$ after light-shock pairings compared with control groups (Fig. 4; exon I, $F_{(2,13)}=6.9, p=0.009$; exon III, $\left.F_{(2,12)}=27, p<0.001\right)$. Levels of exon II $\left(F_{(2,12)}=1.1\right.$, $p=0.4)$ and exon IV $\left(F_{(2,12)}=0.15, p=0.86\right)$ transcripts did not appear to be changing subsequent to fear conditioning. These results indicate that only a subset of the BDNF transcripts are increasing following associative fear conditioning.

In experiments using the exon $\mathrm{V}$ BDNF probe as well as the four specific exon probes (Figs. 1D and 4), we find no significant increases in transcripts in groups receiving the shock alone compared with light alone during training (all $p>0.35$ ). As shown in Figure $1 C$, this type of presentation does not result in significant cue-specific learning. Furthermore, as has been shown in other experiments with significant pre-exposures to the context, we find no enhancement of the baseline startle within this context, 

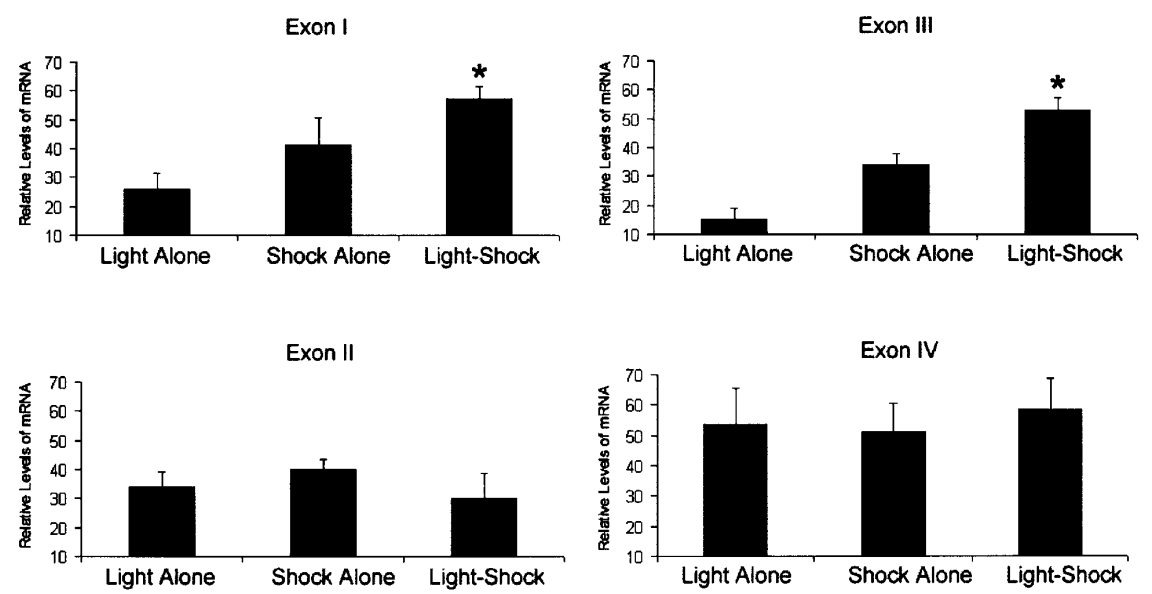

Figure 4. Changes in levels of BDNF transcripts following fear conditioning. Summary of the changes in levels of exons I-IV following light-shock associative fear conditioning or light-alone or shock-alone controls. Gene expression was analyzed $2 \mathrm{~h}$ following training. Levels of transcripts for exon I and exon III are significantly increased in the BLA after light-shock fear conditioning when compared with control groups (* ANOVA; $p \leq 0.05$ ). Levels of exons II and IV are comparable across groups.

suggesting there is no significant contextual fear conditioning (Ressler et al. 2002; Rattiner et al. 2004). Therefore, these experiments demonstrate that the significant increases in BDNF exon transcripts within the BLA, as seen with light-shock pairings, are specific for the process mediating associative learning and not for the attentional, pain, or stress pathways mediated by the shock alone.

We have shown that BDNF exons I, III, and V were transcriptionally up-regulated during the consolidation of fear learning, with no apparent change in BDNF exons II and IV. These results provide the first evidence of differential BDNF promoter regulation or alternative splicing mechanisms in response to a behaviorally relevant learning paradigm. These results are consistent with the current in vitro and nonbehavioral in vivo literature suggesting that expression of transcripts containing exon I and III are most sensitive to activity-dependent regulation and are readily induced in response to synaptic activity (Metsis et al. 1993; Timmusk et al. 1993; Kokaia et al. 1994).

Most studies examining BDNF mRNA levels following various manipulations have ignored the differential usage of promoters and 5 -untranslated exons, and have instead described the combined expression of BDNF. These studies using in situ probes that only recognized exon $\mathrm{V}$, the protein-coding region of the BDNF gene, have revealed marked increases in BDNF mRNA after hippocampally dependent learning and memory tasks (Timmusk et al. 1993). Presumably these increases are the sum result of the differential regulation of BDNF exons I-IV. Because they are detecting the entire pool of BDNF transcripts, however, it is impossible to determine which of the transcripts are changing as a result of the manipulation.

Our current findings suggest that the BDNF gene is indeed differentially regulated in the amygdala following fear conditioning. Fear conditioning may engage different cellular mechanisms controlling the activity-dependent regulation of BDNF mRNAs containing exons I and III. Use of these multiple promoters is likely to direct the activity-specific expression of BDNF. Both exon I and III promoters are selectively activated by calcium influx. The exon III promoter contains a calcium-responsive element (CaRE1) that is regulated by a newly discovered calciumresponsive transcription factor, CaRF (Tabuchi et al. 2002; Tao et al. 2002). The exon I promoter contains a cAMP-responsive element that is overlapped by the upstream stimulatory factor (USF) binding element. Both CREB and USF1/ USF 2 transcription factors contribute to the calcium-mediated activation of the exon I promoter (Tabuchi et al. 2002). There is currently little known about the regulatory sequences within the exon II and exon IV promoters, although it has been shown that the nuclear isoforms of CamKII are involved in the expression of the exon IV promoter (Takeuchi et al. 2002).

The significance of this differential regulation is not well understood. It is possible that the only function of differential exon usage is for differential transcriptional control. It is also possible that a functional role of the different exons exists. For example, exons I and III may contain targeting sequences allowing mRNA transport to the dendrites, where it can affect local synaptic plasticity. It has been shown that BDNF mRNA is dendritically targeted (Tongiorgi et al. 1997; Righi et al. 2000); however, it is unknown which of the different BDNF transcripts are targeted to the dendrites or whether the targeting sequences lie in the $5^{\prime}$ - or $3^{\prime}$ untranslated regions. Future experiments examining the differential cellular localization of transcripts in vitro will explore the hypothesis that location and sorting of different transcripts is a function of 5'-exon usage.

Another intriguing possibility is that differential 5'-UTR utilization may partially determine rates of translation or mRNA stability. An increasing literature suggests that the 5'-UTRs of some dendritically translated mRNAs allow for more efficient translation via an internal ribosomal entry site (IRES) that allows initiator-independent translation (Gromeier et al. 1999; Jopling and Willis 2001; Pinkstaff et al. 2001). There are few consensus sites for IRES function, and it is not yet clear if BDNF contains such sequences, but future studies examining these possibilities are warranted. Of note, a recent preliminary report suggests that an untranslated exon of the human BDNF gene may contain an IRES function (Zaitsev and Lu 2003).

In summary, these data indicate differential promoter control or splicing of the BDNF gene following fear conditioning in the amygdala. It would be important to know whether similar transcriptional control of BDNF is found in other behavioral learning paradigms. Further research on the role of this mechanism of regulation should be informative, both from the perspective of transcriptional control of dynamic levels of BDNF protein, as well as for the differential functions of the 5 '-untranslated mRNA transcripts in vivo.

\section{Acknowledgments}

This research was supported by a Rockefeller Brothers Fund Culpeper Scholarship in Medical Sciences, the Science and Technology Center Program (Center for Behavioral Neuroscience) of the National Science Foundation under Agreement IBN-987675, and grants from the National Institute of Health $(\mathrm{MH} 47840, \mathrm{MH}$ 59906, and MH69884).

\section{References}

Aliaga, E., Arancibia, S., Givalois, L., and Tapia-Arancibia, L. 2002.

Osmotic stress increases brain-derived neurotrophic factor messenger RNA expression in the hypothalamic supraoptic nucleus with differential regulation of its transcripts. Relation to arginine-vasopressin content. Neuroscience 112: 841-850.

Berchtold, N.C., Oliff, H.S., Isackson, P., and Cotman, C.W. 1999. 
Hippocampal BDNF mRNA shows a diurnal regulation, primarily in the exon III transcript. Brain Res. Mol. Brain Res. 71: 11-22.

Bishop, J.F., Mueller, G.P., and Mouradian, M.M. 1994. Alternate 5' exons in the rat brain-derived neurotrophic factor gene: Differential patterns of expression across brain regions. Brain Res. Mol. Brain Res. 26: $225-232$.

Cassella, J.V., Harty, T.P., and Davis, M. 1986. Fear conditioning, pre-pulse inhibition and drug modulation of a short latency startle response measured electromyographically from neck muscles in the rat. Physiol. Behav. 36: 1187-1191.

Davis, M., Falls, W.A., Campeau, S., and Kim, M. 1993. Fear-potentiated startle: A neural and pharmacological analysis. Behav. Brain Res. 58: $175-198$

Dias, B.G., Banerjee, S.B., Duman, R.S., and Vaidya, V.A. 2003. Differential regulation of brain derived neurotrophic factor transcripts by antidepressant treatments in the adult rat brain. Neuropharmacology 45: 553-563.

Fanselow, M.S. and LeDoux, J.E. 1999. Why we think plasticity underlying Pavlovian fear conditioning occurs in the basolateral amygdala. Neuron 23: 229-232.

Figurov, A., Pozzo-Miller, L., Olafsson, P., Wang, T., and Lu, B. 1996. Regulation of synaptic responses to high-frequency stimulation and LTP by neurotrophins in the hippocampus. Nature 381: 706-709.

Gromeier, M., Bossert, B., Arita, M., Nomoto, A., and Wimmer, E. 1999 Dual stem loops within the poliovirus internal ribosomal entry site control neurovirulence. J. Virol. 73: 958-964.

Hall, J., Thomas, K.L., and Everitt, B.J. 2000. Rapid and selective induction of BDNF expression in the hippocampus during contextual learning. Nat. Neurosci. 3: 533-535.

Jopling, C.L. and Willis, A.E. 2001. N-myc translation is initiated via an internal ribosome entry segment that displays enhanced activity in neuronal cells. Oncogene 20: $2664-2670$.

Kang, H., Welcher, A.A., Shelton, D., and Schuman, E.M. 1997. Neurotrophins and time: Different roles for TrkB signaling in hippocampal long-term potentiation. Neuron 19: 653-664.

Kokaia, Z., Metsis, M., Kokaia, M., Bengzon, J., Elmer, E., Smith, M.L., Timmusk, T., Siesjo, B.K., Persson, H., and Lindvall, O. 1994. Brain insults in rats induce increased expression of the BDNF gene through differential use of multiple promoters. Eur. J. Neurosci. 6: $587-596$.

Korte, M., Carroll, P., Wolf, E., Brem, G., Thoenen, H., and Bonhoeffer, T. 1995. Hippocampal long-term potentiation is impaired in mice lacking brain-derived neurotrophic factor. Proc. Natl. Acad. Sci. 92: 8856-8860.

Lindvall, O., Ernfors, P., Bengzon, J., Kokaia, Z., Smith, M.L., Siesjo, B.K., and Persson, H. 1992. Differential regulation of mRNAs for nerve growth factor, brain-derived neurotrophic factor, and neurotrophin 3 in the adult rat brain following cerebral ischemia and hypoglycemic coma. Proc. Natl. Acad. Sci. 89: 648-652.

Liu, Y., Fowler, C.D., and Wang, Z. 2001. Ontogeny of brain-derived neurotrophic factor gene expression in the forebrain of prairie and montane voles. Brain Res. Dev. Brain Res. 127: 51-61.

Metsis, M., Timmusk, T., Arenas, E., and Persson, H. 1993. Differential usage of multiple brain-derived neurotrophic factor promoters in the rat brain following neuronal activation. Proc. Natl. Acad. Sci. 90: 8802-8806.

Oliff, H.S., Berchtold, N.C., Isackson, P., and Cotman, C.W. 1998 Exercise-induced regulation of brain-derived neurotrophic factor (BDNF) transcripts in the rat hippocampus. Brain Res. Mol. Brain Res.
61: $147-153$.

Paschall, G.Y. and Davis, M. 2002. Olfactory-mediated fear-potentiated startle. Behav. Neurosci. 116: 4-12.

Pelletier, J., Counis, R., de Reviers, M.M., and Tillet, Y. 1992. Localization of luteinizing hormone $\beta$-mRNA by in situ hybridization in the sheep pars tuberalis. Cell Tissue Res. 267: 301-306.

Pinkstaff, J.K., Chappell, S.A., Mauro, V.P., Edelman, G.M., and Krushel, L.A. 2001. Internal initiation of translation of five dendritically localized neuronal mRNAs. Proc. Natl. Acad. Sci. 98: 2770-2775.

Poo, M.M. 2001. Neurotrophins as synaptic modulators. Nat. Rev. Neurosci. 2: 24-32.

Poulsen, F.R., Lauterborn, J.C., Zimmer, J., and Gall, C.M. 2004. Differential expression of brain-derived neurotrophic factor transcripts after pilocarpine-induced seizure-like activity is related to mode of $\mathrm{Ca}^{2+}$ entry. Neuroscience 126: 665-676.

Rattiner, L.M., Davis, M., French, C.T., and Ressler, K.J. 2004. BDNF and TrkB receptor involvement in amygdala dependent fear conditioning. J. Neurosci. 24: 4796-4806.

Remick, D.G., Scales, W.E., May, M.A., Spengler, M., Nguyen, D., and Kunkel, S.L. 1988. In situ hybridization analysis of macrophage-derived tumor necrosis factor and interleukin-1 mRNA. Lab. Invest. 59: 809-816.

Ressler, K.J., Paschall, G., Zhou, X.L., and Davis, M. 2002. Regulation of synaptic plasticity genes during consolidation of fear conditioning. $J$. Neurosci. 22: 7892-7902.

Righi, M., Tongiorgi, E., and Cattaneo, A. 2000. Brain-derived neurotrophic factor (BDNF) induces dendritic targeting of BDNF and tyrosine kinase B mRNAs in hippocampal neurons through a phosphatidylinositol-3 kinase-dependent pathway. J. Neurosci. 20: $3165-3174$.

Tabuchi, A., Sakaya, H., Kisukeda, T., Fushiki, H., and Tsuda, M. 2002. Involvement of an upstream stimulatory factor as well as cAMP-responsive element-binding protein in the activation of brain-derived neurotrophic factor gene promoter I. J. Biol. Chem. 277: 35920-35931.

Takeuchi, Y., Miyamoto, E., and Fukunaga, K. 2002. Analysis on the promoter region of exon IV brain-derived neurotrophic factor in NG108-15 cells. J. Neurochem. 83: 67-79.

Tao, X., West, A.E., Chen, W.G., Corfas, G., and Greenberg, M.E. 2002. A calcium-responsive transcription factor, $\mathrm{CaRF}$, that regulates neuronal activity-dependent expression of BDNF. Neuron 33: 383-395.

Timmusk, T., Palm, K., Metsis, M., Reintam, T., Paalme, V., Saarma, M., and Persson, H. 1993. Multiple promoters direct tissue-specific expression of the rat BDNF gene. Neuron 10: $475-489$.

Tongiorgi, E., Righi, M., and Cattaneo, A. 1997. Activity-dependent dendritic targeting of BDNF and TrkB mRNAs in hippocampal neurons. J. Neurosci. 17: 9492-9505.

Walker, D.L. and Davis, M. 2000. Involvement of N-methyl-D-aspartate (NMDA) receptors within the amygdala in short- versus long-term memory for fear conditioning as assessed with fear-potentiated startle. Behav. Neurosci. 114: 1019-1033.

Zaitsev, E. and Lu, B. 2003. Cap-independent translation of BDNF: IRES activity of two BDNF RNA transcripts. Soc. Neurosci. Abstr. Program No. 334.5

Received May 15, 2004; accepted in revised form August 10, 2004. 


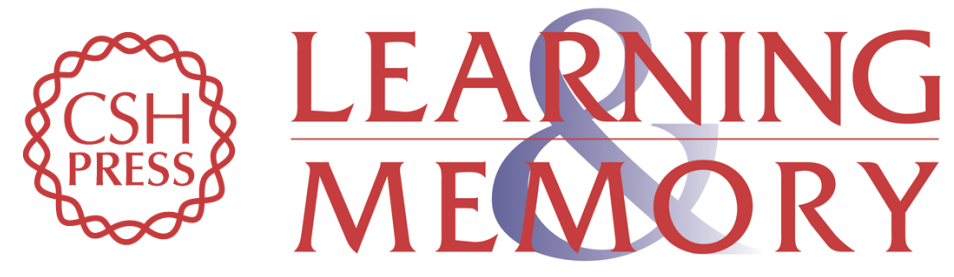

\section{Differential regulation of brain-derived neurotrophic factor transcripts during the consolidation of fear learning}

Lisa M. Rattiner, Michael Davis and Kerry J. Ressler

Learn. Mem. 2004, 11:

Access the most recent version at doi:10.1101//m.83304

References This article cites 33 articles, 10 of which can be accessed free at: http://learnmem.cshlp.org/content/11/6/727.full.html\#ref-list-1

License

Email Alerting Receive free email alerts when new articles cite this article - sign up in the box at the Service top right corner of the article or click here. 